\title{
Occult pulmonary thromboembolism presenting as diffuse interstitial pneumonitis cancer patients
}

\begin{abstract}
S. W. NEEDLEMAN*
M.D.

\author{
J. O. ARMitAGE \\ M.D.
}

\section{Summary}

The prompt and accurate recognition of non-malignant complications is of critical importance in the care of cancer patients. Pulmonary thromboembolism is particularly important because it is common, treatable and frequently difficult to diagnose. Two patients are presented who died of recurrent pulmonary thromboemboli which were unrecognized because open lung biopsies showed diffuse interstitial pneumonitis. The association of pulmonary thromboembolism and interstitial inflammation has been recognized at autopsy, and there are a number of plausible mechanisms which could link these processes. Interstitial pneumonitis should be added to the numerous protean manifestations with which pulmonary thromboembolism is associated.
\end{abstract}

C. A. ASCHENBRENER

M.D.

†Departments of Internal Medicine and Pathology, University of Iowa Hospitals, Newton Road, Iowa City, $\overrightarrow{\vec{H}}$ 52242, U.S.A.

KEY WORDS: pulmonary embolism, diffuse interstitial pneumonitis, inflammation, thrombosis, cancer chemotherapy.

\section{Introduction}

Diffuse interstitial pneumonitis can acutely complicate the course of patients with a variety of neoplasms. Numerous aetiologies have been reported including fungal infection (Fisher and Armstrong, 1977), Pneumocystis carinii, cytomegalovirus and other viral infections (Singer et al., 1979), radiation therapy (Wara, Phillips and Margolis, 1973), hypersensitivity to a variety of anti-neoplastic agents such as bleomycin (Whitcomb, 1973), and graft-versushost disease (Graze and Gale, 1979). Pulmonary thromboembolism has been reported to be associated with prominent interstitial fibrosis at autopsy (Saldeen, 1979), but this association is not clinically

\footnotetext{
*Present address: Laboratory of Celluar and Molecular Biology, National Institutes of Health, National Cancer Institute, Building 37, Room 1C20, Bethesda, Maryland 20205, U.S.A.
}

recognized. We have recently observed 2 cases of interstitial pneumonitis in patients with cancer whom we feel occult, underlying pulmonary thronep boembolism was the aetiology.

\section{Case reports}

Case 1

A 65-year-old Caucasian female presented plaining of weakness. Physical examination disciseg a large abdominal mass. Laboratory investigation revealed an autoimmune haemolytic anaemia. Pre nisone was begun, with improvement of the anaemis, and she was admitted to the hospital for laparotom 8 . At this procedure, an extensive retroperitoneal malig nancy was demonstrated, and biopsy of lymph nodgs led to the diagnosis of diffuse histiocytic lymphoma. Her post-operative course was uneventful and she was subsequently begun on combination chemg therapy comprising cyclophosphamide, adriamycin, vincristine and prednisone.

During the second chemotherapy cycle, she con? plained of fever, weakness, dyspnoea and productize cough and she was readmitted to the hospital. O examination, she was febrile and the chest was cleag. The $\mathrm{PO}_{2}$ on room air was $53 \mathrm{mmHg}$, while the $\mathrm{PcQ}$ was $26 \mathrm{mmHg}$ and $\mathrm{pH} \mathrm{7.53.} \mathrm{Chest} \mathrm{X-ray} \mathrm{initials}$ revealed interstitial infiltrates of the left upper lobe which rapidly progressed to involve all lung fields? Diffuse dry rales became audible. Fever persisted despite cephalosporin therapy. Cultures of blood urine and sputum were negative.

Fibreoptic bronchoscopy was performed and tê vealed an inflamed tracheobronchial tree. A trans bronchial lung biopsy revealed interstitial inflammo? tion and fibrosis. She subsequently developed venti latory failure. An open lung biopsy was performed 
and demonstrated interstitial pneumonitis. Corticosteroids in high doses and antituberculous therapy were begun. Her oxygen tension stabilized and she was discharged. Fever and dyspnoea recurred however, and several weeks later she developed an acute dyspnoeic episode and died.

At autopsy, the left lung exhibited an apical abscess, $3.5 \mathrm{~cm}$ in diameter; numerous wedge-shaped organized infarcts were scattered throughout both lungs. The right and left main pulmonary arteries were occluded by recent antemortem thromboemboli. On microscopic examination, there were diffuse interstitial inflammatory changes and fibrosis. Numerous small arteries and arterioles were occluded with emboli, although in the most peripheral vessels they were less prominent. Thrombi were found in the thigh veins bilaterally. Post-mortem lung and blood cultures were negative.

\section{Case 2}

A 34-year-old Caucasian female presented with a 48-hr history of easy bruising, gross haematuria and malaise. Circulating leukaemic cells were observed and she was referred for further evaluation.

The white cell count was elevated at $18.0 \times 10^{9} /$ litre with $14 \times 10^{9} /$ litre blasts and $0.72 \times 10^{9} /$ litre segmented neutrophils. Haemoglobin was $9 \mathrm{~g} / \mathrm{dl}$ with normal erythrocyte indices. The platelets were decreased at $5 \times 10^{9} /$ litre. The fibrinogen was $82 \mathrm{mg} / \mathrm{dl}$ and the prothrombin time was prolonged to $17 / \mathrm{s}$. Fibrin degradation products were markedly elevated. Marrow examination revealed a hypercellular marrow packed with myeloblasts and promyelocytes, with occasional auer rods. Remission induction was attempted with thioguanine, cytarabine, daunomycin, vincristine and prednisone. Heparin and cryoprecipitate were administered for the coagulopathy.

On the fourth hospital day, fever, acute dyspnoea, hypoxaemia, tachycardia, tachypnoea, diffuse rales and pulmonary infiltrates (Fig. 1) were noted. Antibiotics were begun, heparin continued, and the respiratory distress abruptly resolved. Laboratory evidence of disseminated intravascular coagulation (DIC) responded to heparin administration. Similar episodes occurred on the 12th, 13th, 18th and 20th days. Trimethoprim-sulphamethoxazole was added, but the fever persisted. On the 23rd day, an open lung biopsy was performed and revealed alveolar septal fibrosis, reactive proliferation of alveolar pneumocytes, and residual proteinaceous debris in alveoli and small bronchioles. Inflammatory cells were evident in the alveolar septae. Special stains for bacteria, mycobacteria, and fungi were negative, as were viral cultures and direct tissue immunofluores- cence for Legionella. The small arteries and veins were devoid of thrombi.

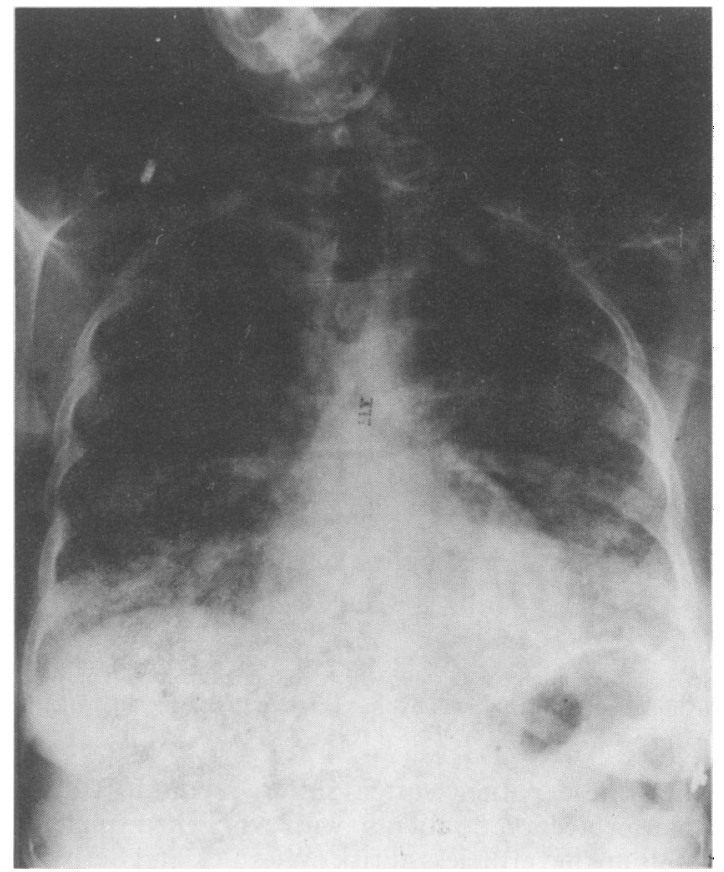

FIG. 1. Chest X-ray, P/A projection, Case 2, 4th hospital day. Diffuse patchy interstitial infiltrates are noted.

Subsequently, the patient suffered acute renal failure, a second central nervous system haemorrhage, cardiopulmonary arrest, and died on the 30th hospital day. At autopsy, the lungs had a nodular appearance produced by firm, raised, deep red infarcts scattered through the parenchyma. The infarcts were associated with visible, often friable thrombi in medium and large vessels and were predominantly central, rather than in the classic subpleural location. On microscopic examination, most of the lung tissue appeared similar to the biopsy specimen and was similarly devoid of visible bacteria, mycobacteria, and fungi. Centrally, however, there were numerous pulmonary infarcts, associated with arterial and venous thrombi which consisted of masses of fibrin and fungal hyphae. Special stains demonstrated vascular mural invasion by fungi in occasional large vessels (Fig. 2).

\section{Discussion}

Pulmonary embolism is a highly treatable disease with protean manifestations. If unrecognized, the outcome can frequently be fatal. Pulmonary thromboembolism complicates the course of cancer patients frequently. Although thrombocytopaenia, 


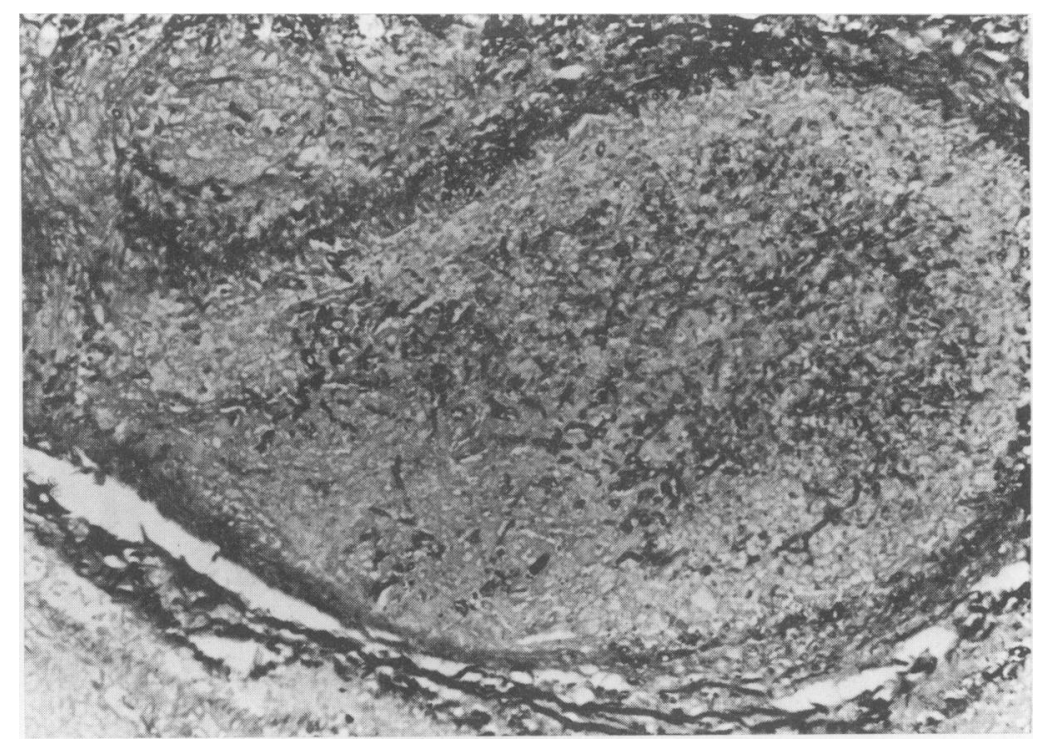

FIG. 2. Large pulmonary artery which contains thrombus composed of packed fibrin and dichotomous hyphae consistent with Aspergillus. 马ीt $_{3}$ the upper edge of the vessel, hyphae are seen to completely penetrate the vessel wall, extending into the adjacent infarct ( $\times 66-G$ rocott stain).

which these patients often suffer, seems to confer some protection, patients with very low platelet counts are nevertheless at risk (Wiernick and Serpick, 1969; Needleman, Stein and Hoak, 1981). Thus, a high index of suspicion for pulmonary embolus must be maintained by the clinician in the care of the cancer patient.

In recent years, many of the cellular and molecular phenomena which initiate and regulate inflammation or thrombosis have been shown to be interrelated. A number of mediators of platelet adhesion and aggregation (e.g. ADP, epinephrine) potentiate leucocyte adhesion; conversely, non-steroidal anti-inflammatory agents inhibit platelet aggregation as well as leukocyte adhesion. The platelet can be a source of lysozomal enzymes which are important in inflammation, as well as leucocyte chemotactic factors such as hydroxyeicosatetraenoic acid (HETE); moreover, in certain situations, the leucocyte releases procoagulant material, a process which is, in turn, enhanced by platelet divided lipids (Needleman and Hoak, 1982). Study of the plasma proteins as well, have yielded potential interfaces of thrombosis and inflammation, such as activation of complement, Hageman factor, Fletcher factor and others (Ratnoff, 1980). The precise relationship of these patients' thromboemboli to their development of diffuse interstitial pneumonitis remains unclear. However, it is tempting to speculate that the microthrombi in the small interstitial vessels might be related to interstitial inflammation through activation of both processes at one or more of these interfaces.
The possibility that these were 2 independe processes involving different regions of the lungs must be addressed, in view of lung biopsies in patient which failed to establish the diagnosis of

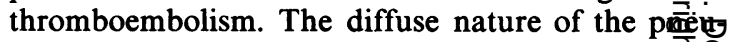
monitis on X-ray as well as the extensive diffrie fibrosis at autopsy mitigate against topograph separation of the processes. Rather, it seems that in the lung periphery where biopsies are performed, the thromboemboli involve microscopic vascular struc tures, and are themselves miniscule. The minue emboli are probably the most readily resolved by fibrinolytic enzymes. Thus, it seems that open lun biopsy in these patients might examine a portion of lung where resolution of the thromboembolis precedes that of interstitial inflammation.

It is also conceivable that the cytolytic chem therapy, which both patients received, contributed to the development of diffuse interstitial pneumonitis. While none of the drugs given have been firmby associated with interstitial pneumonitis, one cann git exclude the possibility that any of the drugs, alone or in combination could give rise to inflammation as result of direct toxicity to vascular endothelium idiosyncratic hypersensitivity. In addition, neutroo penia induced by these drugs could predispose to thrombosis by removing the fibrinolytic and $/$ f anticoagulant activities of granulocytes from the blood (Needleman et al., 1981).

A significant number of thrombopenic patien. who suffer thromboembolism will have occult fungat infection as did Case 2 (Needleman et al., 1981). 
Many fungi are heavily endowed with proteolytic capacities which can directly cause both inflammation and thrombosis. Alternatively, the ability of these organisms to invade vessel walls and adjacent tissue (Fig. 2), can cause lysozomal enzymes and tissue thromboplastin to be released from the tissue, initiating both processes (Needleman et al., 1981).

The treatment of neoplastic disease depends heavily on the quality and diligence or supportive care in preventing and recognizing non-malignant complications. The importance of pulmonary embolism in this regard can not be overemphasized. The clinician's index of suspicion should not be too greatly diminished by the presence of thrombocytopenia, even if severe (Wiernick and Serpick, 1969; Needleman et al., 1981). Diagnosis can be difficult, owing to the numerous, non-specific manifestations of the disease. These cases suggest that a lung biopsy diagnostic of diffuse interstitial pneumonitis should be added to the wide variety of findings which can be observed in patients with pulmonary embolism.

\section{References}

FISHER, B.D. \& ARMSTRONG, D. (1977) Cryptococcal interstitial pneumonia: value of antigen determination. New England Journal of Medicine, 297, 1440.

GRAZE, P.R. \& GALE, R.P. (1979) Chronic graft versus host disease: a syndrome of disordered immunity. American Journal of Medicine, 66, 611 .

NeEDlemAN, S.W. \& HoAK, J.C. (1982) The role of the platelet and leukocyte in thrombosis. In: Hemostasis and Thrombosis, (Ed. R. Colman), Ch. 51. Lipincott Co., Philadelphia.

Needleman, S.W., Stein, M.M. \& Hoak, J.C. (1981) Pulmonary embolism in patients with acute leukemia and severe thrombocytopenia. Western Journal of Medicine, 135, 9.

RATNOFF, O.D. (1980) A quarter century with Mr Hageman. Thrombosis and Haemostasis, 43, 95.

SALDEEN, T. (1979) The microembolism syndrome. Microvascular Research, 11, 227.

Singer, C., Armstrong, D., Rosen, P.P., Walter, P.D. \& Yu, B. (1979) Diffuse pulmonary infiltrates in immunosuppressed patients. American Journal of Medicine, 66, 110.

WARA, W.M., PhILliPS, T.L. \& MARGolis, L.W. (1973) Radiation pneumonitis: a new approach to the derivation of time-dose factors. Cancer, 32, 547.

WhiтсомB, M.E. (1973) Drug induced lung disease. Chest, 63, 418.

WiernicK, P.H. \& SERPICK, A.A. (1969) Pulmonary embolus in acute myelocytic leukemia. Cancer, 24, 581 .

(Accepted 25 November 1982) 